\title{
BIM FROM LASER CLOUDS AND FINITE ELEMENT ANALYSIS: COMBINING STRUCTURAL ANALYSIS AND GEOMETRIC COMPLEXITY
}

\author{
L. Barazzetti, F. Banfi, R. Brumana, G. Gusmeroli, D. Oreni, M. Previtali, F. Roncoroni, G. Schiantarelli \\ Gicarus Lab, ABC Department, Politecnico di Milano, Piazza Leonardo da Vinci 32, Milan, Italy \\ (luigi.barazzetti, fabrizio.banfi, raffaella.brumana, daniela.oreni, mattia.previtali, fabio.roncoroni)@ polimi.it \\ (gaia.gusmeroli, giuseppe.schiantarelli)@ mail.polimi.it \\ http://www.gicarus.polimi.it
}

\section{Commission V}

KEY WORDS: BIM, Finite Element Analysis, Laser Scanning, Photogrammetry

\begin{abstract}
:
This paper describes the use of BIM models derived from point clouds for structural simulation based on Finite Element Analysis (FEA). Although BIM interoperability has reached a significant level of maturity, the density of laser point clouds provides very detailed BIM models that cannot directly be used in FEA software. The rationalization of the BIM towards a new finite element model is not a simple reduction of the number of nodes. The interconnections between the different elements and their materials require a particular attention: BIM technology includes geometrical aspects and structural considerations that allow one to understand and replicate the constructive elements and their mutual interaction. The information must be accurately investigated to obtain a finite element model suitable for a complete and detailed structural analysis. The aim of this paper is to prove that a drastic reduction of the quality of the BIM model is not necessary. Geometric data encapsulated into dense point clouds can be taken into consideration also for finite element analysis.
\end{abstract}

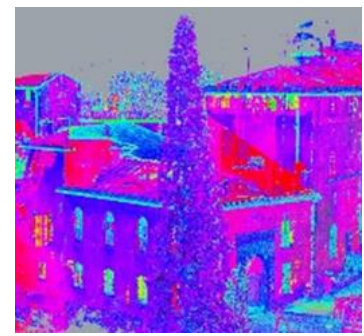

(a)

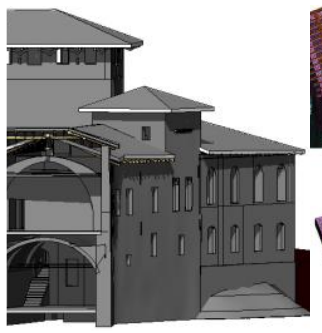

(b)

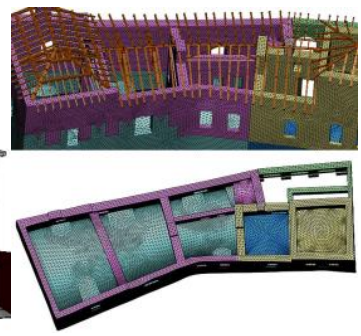

(c)

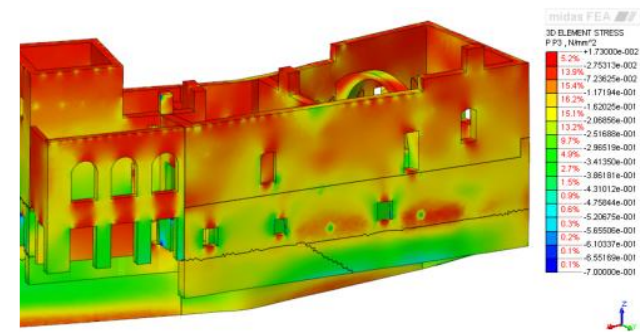

(d)

The phases of the work: (a) point cloud acquisition/registration, (b) creation of the BIM from point clouds by preserving geometric complexity, (c) generation of the finite element model with tetrahedral meshes, (d) structural analysis with the new model. The case study is Castel Masegra, a castle in Sondrio (Italy).

\section{INTRODUCTION}

This aim of this work was to investigate the possibility to transform an accurate BIM of a historical building (HBIM) into a finite element model (FEM, Zienkiewicz, 1971). The starting point of the work is a set of dense laser scanning point clouds able to capture the geometric complexity of the object. The approach is not the typical structural simulation based on simplified models that fit the requirements of software packages for finite element analysis (FEA).

The recent advances in BIM technology led to an incredible interest towards new efficient methodologies in the construction industry, starting from the survey of the object up to the design and management phases (Osello et al., 2012).

Although specific BIM packages with FEA tools are available on the commercial market, they were developed for new buildings with simple and regular objects. A detailed BIM for architectural purposes (derived from point clouds) is much more complex and must be rigorously discretized through a simplification of its geometry, obtaining a new alveolar mesh that tries to preserve the original complexity (Gusmeroli and Schiantarelli, 2014).

The modification of the original BIM is not intended as a simple reduction of the number of nodes. The interconnection between the different parts requires a particular attention. BIM modelling for FEA includes geometrical aspects along with structural considerations that allow one to replicate the constructive elements and their mutual interaction. Geometric and semantic data contained in the architectural model should be accurately rationalized to obtain a new finite element model for structural analysis.

The aim of this paper is to prove that a drastic reduction of the of the model is not necessary. The geometry encapsulated into dense point clouds can be accurately taken into consideration also for FEA. Complex elements (such as vaults) and structural anomalies (e.g. verticality) are turned into 3D surfaces by an intelligent node-to-node connection, obtaining models that can be managed for static analysis. This is feasible not only for 
modern buildings with predefined mathematical shapes, but also for complex historical objects with several irregularities.

The case study presented in this work is Castel Masegra, a castle located in Sondrio (Italy). A detailed historical BIM was derived from laser scanning point clouds (ca 7 billion points). Building information modelling was carried out by dividing the different structural objects and their constructive logic. Chronological, material, and stratigraphic aspects were also taken into account. This step is not only useful for architectural purposes, but also for further static and dynamic simulations where the temporal evolution of the castle provides additional data about its logic of constrctions.

The historical BIM was then re-adapted for structural purposes obtaining a new sub-model with more details than those usually used for this kind of analysis. A set of tetrahedral meshes was generated to guarantee an adequate representation of the stress/strain distribution. Several problems were taken into consideration, such as the node-to-node correspondence (compatibility of the mesh), very distorted or small elements, and local imprecisions. These effects are extremely important and should be removed to obtain a consistent model for FEA. In fact, small errors and local inconsistencies lead to a failure during the simulation, as the continuity of the model is not guaranteed.

Finally, a linear simulation was carried out in Midas-FEA, a package for finite element analysis. The level of detail of the model allowed one to simultaneously simulate the behaviour of different structural elements (vaults, walls, beams, columns, etc.). The result demonstrates that the creation of a consistent alveolar mesh from the (H)BIM is a crucial step and deserves a special attention.

\section{DATA ACQUISITION AND PROCESSING}

The complexity and size of the castle required a laser scanning surveying, that is a powerful technique to obtain complex reconstructions (Brumana et a., 2014a; Remondino et al., 2008). The instruments used are a Laser Scanner Faro Focus 3D and a total station Leica TS30. A robust geodetic network made up of 68 stations was measured in about 4 days. Least Squares adjustment provided an average point precision of about \pm 1.2 mm (Fig. 2).

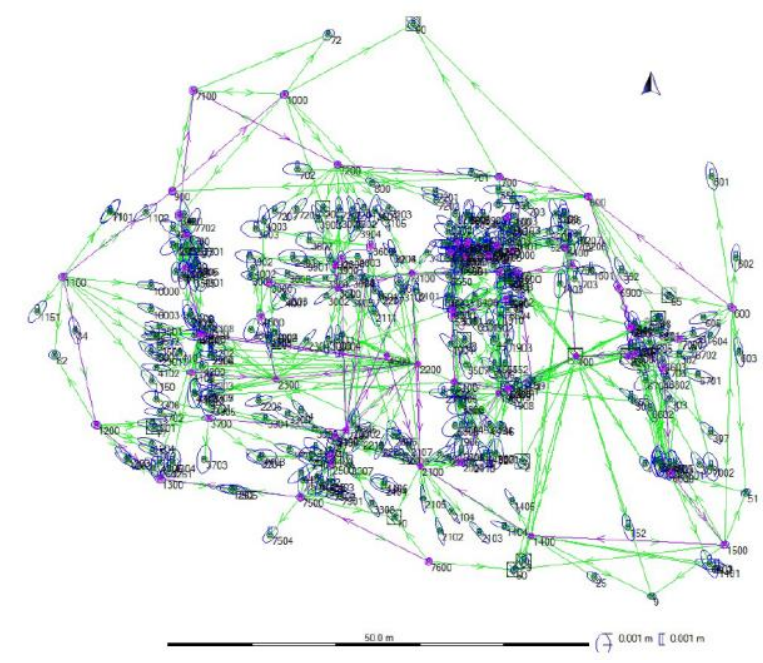

Fig. 2. The geodetic network measured with a total station Leica TS30 (the average precision is $\pm 1.2 \mathrm{~mm}$ ).

The network provides a reference system for scan registration. 176 scans were acquired to obtain a final point cloud made up of 7.5 billion points, which were registered with an average precision of $\pm 3 \mathrm{~mm}$.

Overall, total station and laser data were acquired in less than a week. Then, small integrations were needed to capture occluded areas in the first surveying phase. This proves the level of maturity reached by these instruments. It is clear that the time needed for data processing (especially BIM generation) is much longer because of the level of detail achievable from such huge dataset.

As the goal is a reconstruction for architectural and structural purposes, the surveying phase cannot be limited to the shape (geometry is just one of the elements to be taken into consideration). As the goal is the creation of an interoperable BIM and its distribution among the different operators that work on the castle (engineers, architects, historians, archaeologists, restorers, etc.) the survey must include historical analysis, materials, construction phases, technological aspects, stratigraphic analysis, and information from other inspections such as infrared thermography or structural tests (flat-jacks, coring, etc.) (Binda and Tiraboschi, 1999; Colla et al., 2008; Gregorczyk and Lourenco, 2000; Rosina and Grinzato, 2001).

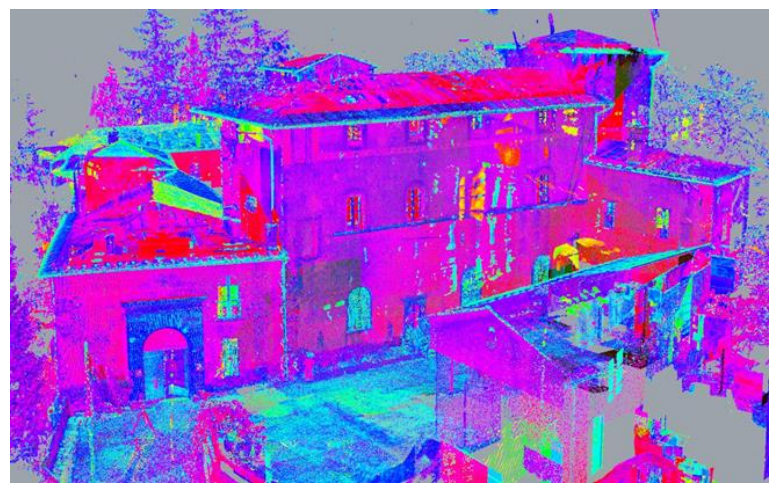

Fig. 3. A visualization of the point cloud after scan registration (176 scans, 7.5 billion points).

\section{GENERATION OF THE BIM MODEL}

\subsection{Why use BIM technology?}

The creation of a detailed BIM is the second step of the project. The use of a BIM instead of a simple 3D model is motivated by the advantages offered by this technology with advanced parametric modelling tools, functional intelligence, and object attributes.

BIM technology simplifies the work of different operators with new interoperability standards: the different operators involved in the project can update and modify the BIM according to their needs. Then, the other operators can immediately evaluate the effect of the change.

The typical inconsistencies of CAD drawings should be limited. A robust exchange of information can limit the creation of additional documents (drawings, reports, ...), towards a better cooperation in the architecture, engineering and construction industry (AEC), where new instruments that allow a consistent exchange of data are required to improve productivity. The interoperability (Drogemuller et al., 2012) has the purpose to connect all the technological aspects involved in construction projects. This is a fundamental requirement not only for a limited number of software packages. Interoperability should be guaranteed for different applications. This approach is extremely important to increase the development of BIM 
oriented projects not only during the design phase, but also for the different phases of the building life cycle.

Although the initial effort for the creation of a BIM is greater than the classical 2D/3D workflows based only on geometry, the advantage of BIM technology becomes effective in the successive phases.

For these reasons, the International Alliance for Interoperability (IAI), today renamed "Building Smart", proposed a new standard open format for the representation of the object in the field of constructions: the Industry Foundation Classes (IFC). Today, IFC $2 \times 3$ files are able to provide a consistent exchange of information between different software. However, the commonly used IFC format was developed for regular shapes, whereas it does not work with complex surfaces. Probably, the new ICF4 will provide additional tools for advanced geometries.

One of the big limitations of software for finite element analysis concerns the low integration with BIM models. It is clear that there is a growing interest towards BIM technology and some software vendors are updating their application to import BIM projects. This confirms that the construction industry is going in the direction of BIM technology.

As things stand at the present, it is rather clear that BIM represents the future of the constructions in a way similar to CAD technology in the past, when manual drawings were progressively substituted by digital representations.

\subsection{BIM from laser clouds: preserving geometric} complexity with advanced modelling tools

Starting from laser data a geometric reconstruction with a high level of detail can be carried out. However, detailed building information modelling cannot simply be carried out with the interpolation of the point cloud with mesh-based algorithms often used in photogrammetry and computer vision. Additional information (e.g. materials, construction stages, stratigraphy, ...) has to be taken into account to create intelligent parametric objects with attributes (Fig. 4).
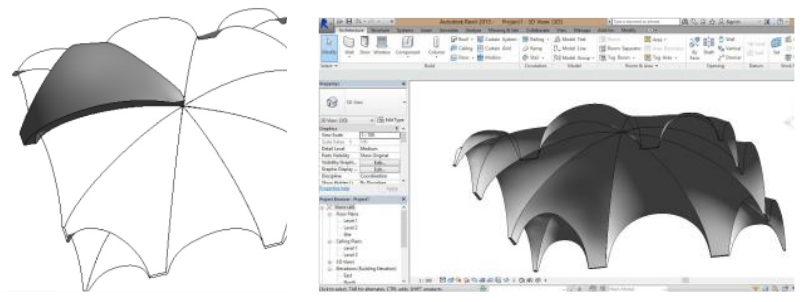

Fig. 4. The vault is a parametric object. The thickness can be set to modify the shape.

An additional problem concerns the lack of parametric software for the management of complex and irregular shapes. BIM software were initially developed to manage new constructions (Eastman et al., 2008; Lee et al., 2006). Building information modelling is usually used for the design, construction, and management phases of new buildings, whereas the use for conservation of historical buildings is still very limited (Fai et al., 2011).

However, they represent an opportunity also for heritage documentation and conservation management, but they still require a methodological discussion and a practical experimentation in order to obtain detailed models of irregular historical objects, that will be really useful for preservation and maintenance activities (Della Torre, 2011; Oreni et al., 2013, 2014a).
The methodology for parametric BIM generation used for the castle is based on a preliminary separation between simple and complex shapes. In the case of simple objects, the tools of most commercial software (Revit or Archicad) can be sufficient. The case of irregular objects (e.g. vaults) is much more complicated. The modelling tools of commercial BIM packages are not adequate to represent the geometric level of detail encapsulated into laser point clouds. For this reason, the procedure described in Oreni et al. (2014b) was used. Vector profiles made up of NURBS curves (Piegl and Tiller, 1997, 1999) provide a curve network for NURBS surfaces, which are then turned into parametric BIM objects.

This methodology was used for the complex shapes found in the castle and it was a very efficient solution to overcome the limitations of BIM software. Some images of the final BIM in Revit are shown in Fig. 5.

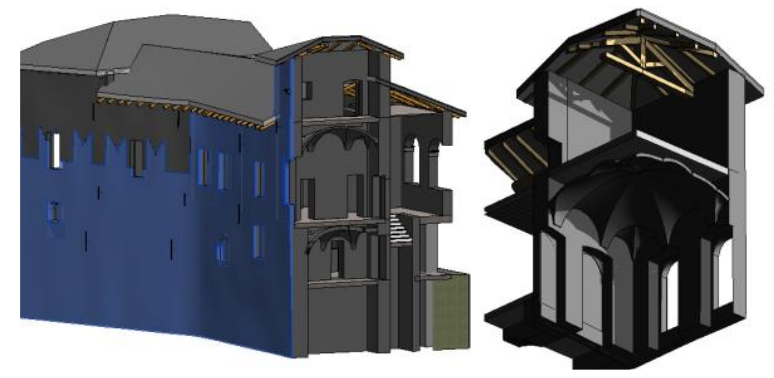

Fig. 5. The parametric BIM in Revit with the advanced representation of irregular objects (e.g. the selected wall features variable verticality issues).

\section{FROM BIM TO FINITE ELEMENTS}

The generated BIM is an interoperable tool that allows different operators to initialize their analyses. Obviously, further data processing could require operations that modify the geometry, not only in terms of different design aspects, but also for specific and advanced simulations requiring simplified models. The aim of this section it to prove that a rationalization can be carried out to transform the BIM model into a tetrahedral mesh for finite element analysis.

\subsection{BIM turned into finite elements}

The finite element method constitutes a general tool for the numerical solution of partial differential equations. The method has a large acceptance in several engineering applications and it is one of the preferred approaches in structural mechanics.

In the finite element method the region of interest is divided into numerous connected sub-regions (or elements) where the solution is obtained from the analysis of shape functions (Strang and Fix, 1973; Zienkiewicz, 1971). The shape function is the function which interpolates the solution between the discrete values obtained at the mesh nodes.

The traditional FEM approaches used in structural analysis rely on simplifications of the elements of a structure into 1D (beams, trusses) or 2D (plates, shells) elements, which are discretized into segments or 2D finite elements.

The integration of 3D finite element analysis in BIM technology is already a concrete possibility for simple and regular buildings. For instance, the structural analysis tool Autodesk Robot Structure is fully integrated with Revit. Different plugins are available to guarantee interoperability with other structural analysis software, such as Midas FEA. These tools allow one (i) 
to transfer the model from Revit and (ii) to obtain a new model for finite element analysis.

The disadvantage of these plugins concern the use of simple load-bearing structures, such as those used for modern buildings with regular columns, beams and walls schematized as $1 \mathrm{D}$ or 2D simple elements.

The tri-dimensional finite element analysis has the possibility to deal with $3 \mathrm{D}$ objects by using a discretization of the structure by means of 3D elements such as tetrahedra or hexaedra. This last options is less used because the number of elements (and consequently the computational effort) is much larger. Moreover, the approximation given by $1 \mathrm{D}$ or $2 \mathrm{D}$ objects in modern structures is more than sufficient in several real projects. However, modern computers allow structural engineers to run complex 3D FEM analysis not only for some particular structural elements, but also extended to the whole structure.

The aim of this work is the re-adaptation of the original BIM to include complex shapes that characterize the load-bearing elements of a historical building (mainly vaults and irregular walls) to arrive at a complete and detailed structural analysis.

This approach is still impossible in a full BIM perspective. The aim was the development of a new procedure able to transform the BIM into a finite element model, without redrawing a new model only for structural analysis.

Generally speaking, the conversion of the objects representing the walls (or other load-bearing elements) could be carried out with auto-meshing algorithms that create the finite element model. On the other hand, this is not a trivial task because of several issues:

- mesh compatibility: single entities need a perfect node-tonode correspondence to ensure geometric continuity between different objects. This result could be automatically achieved with auto-meshing procedures, but the corresponding faces of two generic elements should be exactly the same (same edges): this is not true in the case of complex BIM composed of multiple connected objects;

- local distortions: an ideal mesh must be composed of regular tetrahedral objects. However, the error for the approximation of the solution with an alveolar structure increases with distorted elements. For this reason, very distorted elements should be avoided or at least limited, especially in critical areas;

- small elements: small elements (that increase the level of detail of the BIM) without a direct connection with the load-bearing function should be eliminated to avoid the generation of distorted or small finite elements. This reduces the risk of a large number of small components;

- small imprecisions: the available Revit tools sometimes give very small imprecisions in the final mesh. A finite element analysis requires the exact correspondence of the nodes in order to avoid the generation of thin faces with very distorted "fissure elements";

- complex architectural objects: this is the case of vaults where the parametric modelling capability leads to inconsistent self-intersection between curved objects (e.g. the extrados of a vault modelled from its intrados).

The listed issues are fundamental considerations to obtain an alveolar mesh for FEA. As things stand at the present, there is no software or research algorithm able to transform a complex BIM into a compatible mesh for FEA without approximations.
In other words, the automatic re-adaptation of the historical BIM model towards a consistent mesh required several manual corrections to guarantee a node-to-node continuity that takes into consideration the different elements of the BIM.

The final finite element model (after extensive error removal) is made up 720,393 elements (Fig. 6). Although a simplification of the original shape was still needed, the mesh follows the main irregularities of the structures. In addition, objects with complex geometry (e.g. vaults) did not require excessive simplification.

As mentioned, the aim of this paper is to prove that complex historical BIM created from dense point clouds can be converted into finite element models for structural analysis. As things stand at the present, manual corrections were mandatory but research work can be carried out to develop new data processing algorithms able to consider not only geometrical aspects, but also material properties and logic of construction.
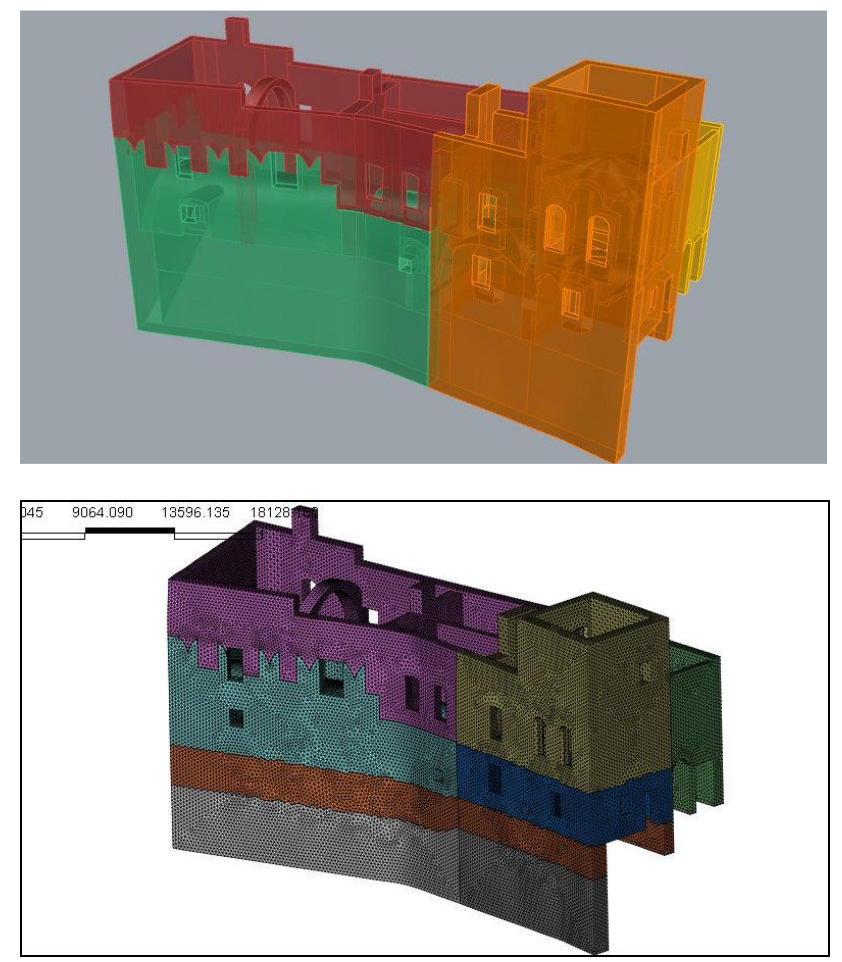

Fig. 6. The BIM turned into a finite element model following construction stages and material properties.

\subsection{The finite element analysis}

The structural analysis can be run after the creation of the finite element model. Input parameters concern the identification of boundary conditions, material properties, and loading conditions. This information can be partially available from the BIM, which is not limited to geometrical aspects.

The simulation carried out is a linear elastic analysis under selfweight. This is a fundamental step before other analysis for the preliminary check of model reliability and gross error removal. Irregular stress information or deformation patterns can be easily recognized under self-weight. Secondly, it allows one to revise and adjust boundary conditions.

As data processing is relatively fast in the case of linear analysis, several simulations can be run to correct setup errors. Different modifications of boundary conditions can be performed to find the most appropriate for the case under investigation. Moreover, the results in terms of stress can be 
directly compared with single-flat jack measurements. It is possible to have a feedback about model reliability in the different areas. Initial hypotheses are progressively adjusted to reach a better consistence with the experimental tests.

Historical information was extremely important to define input parameters. Material properties were assigned according to the stages of construction. The castle was subdivided into several parts by using the historical evolution and the state of conservation of the materials. Material data were also integrated with other destructive and non-destructive techniques able to distinguish areas with good materials from those with bad materials. This is a fundamental point for historical structures that could reveal a high variability of the same parameter.

The self-weight of the load-bearing elements can be automatically computed from the model (Fig. 7). The dead loads of the elements excluded from the model were applied to the structure as distributed pressure loads. Examples of elements directly converted into dead loads are infilling materials on the vaults and wooden slabs.

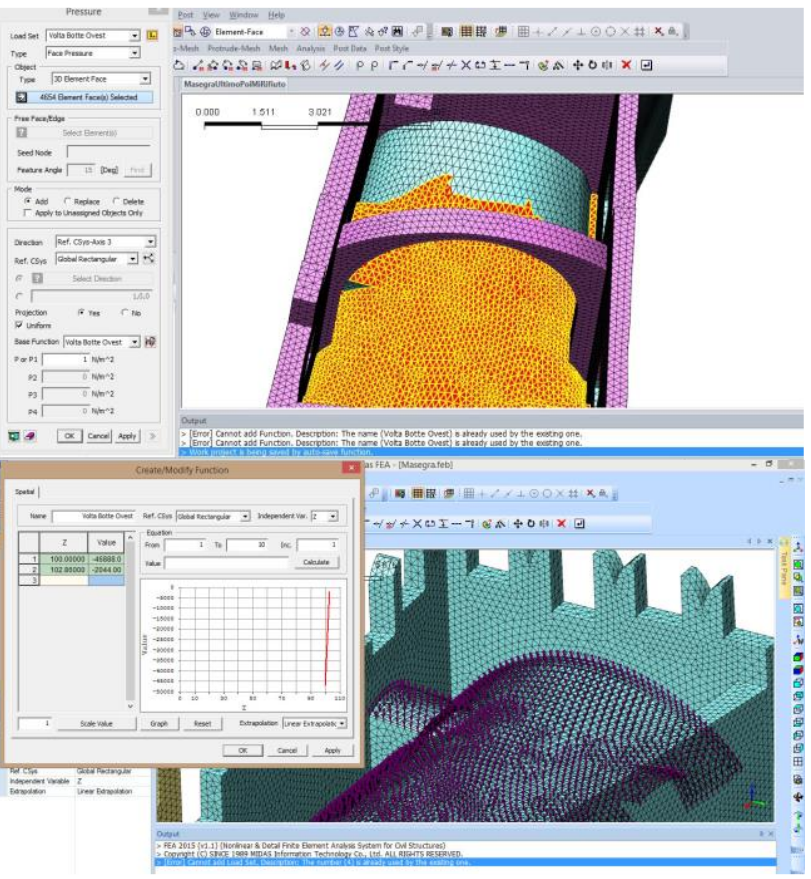

Fig. 7. Assignment of dead load on a barrel vault by using the faces of the mesh.

A correct setup of boundary conditions is one of the most important issues. The castle raises on a cliff and its different parts have different foundations. Some walls are directly connected to the rock, whereas others have superficial foundations. The information concerning foundation typologies was obtained from both coring and visual inspection. Additional parameters were available from the work carried out by the Municipality of Sondrio, which performed previous restoration work in the castle. However, in some cases the information was completely missing and some hypotheses were derived by considering the conditions of the surrounding areas.

Shown in Fig. 8 are the general results obtained in terms of stress (for the whole South wing of the castle). The colour ramp of the stress-plot was adjusted to remove the local influence of stress concentration for the foundations. The value of stress distribution is lower than the ultimate strength of masonry estimated by jack tests. Therefore, no particular problem in terms of resistance is expected.
More exhaustive analyses are currently in progress to check the quality of the obtained results. On the other hand, our preliminary solutions have a direct connection to some local damages found in the structure (e.g. cracks).
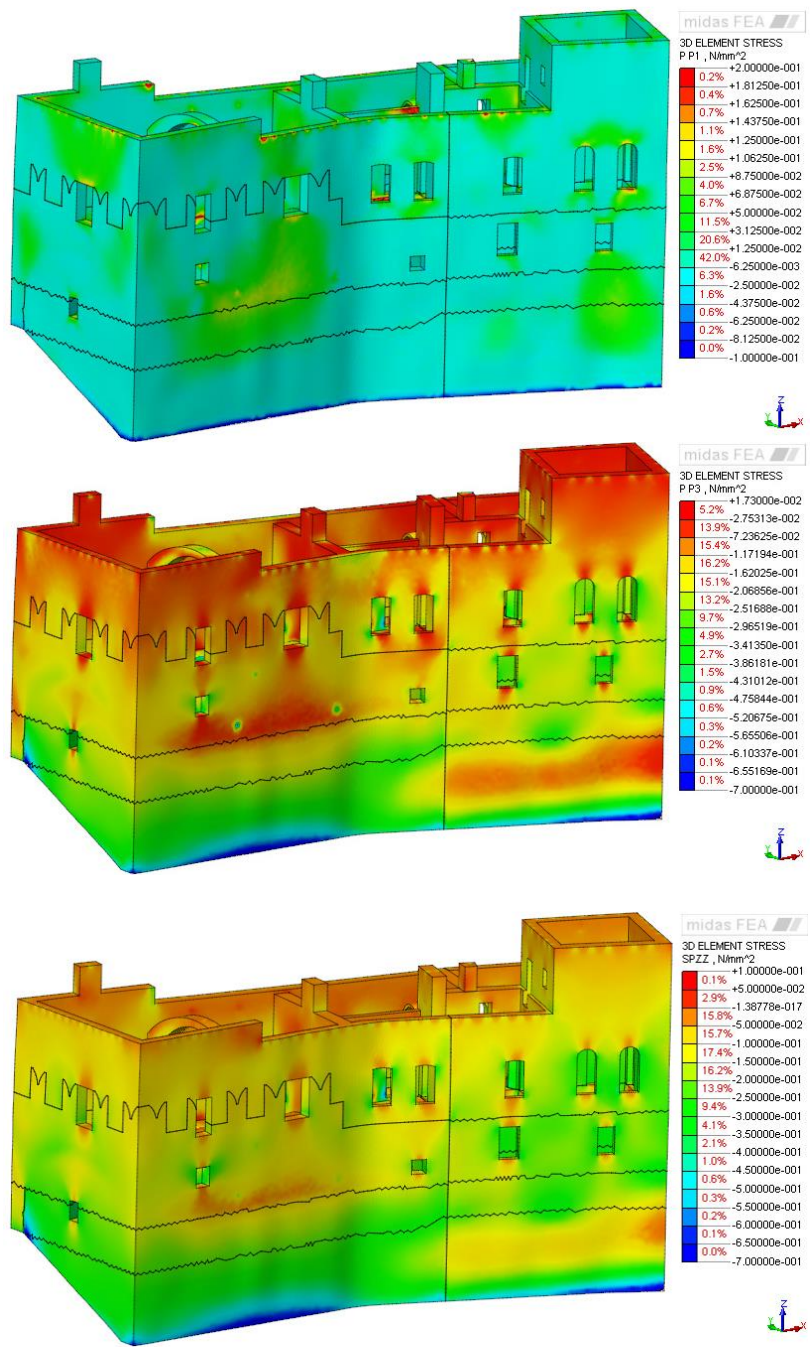

Fig. 8. Stress after simulation via alveolar mesh derived from BIM: (top) first principal stress; (middle) third principal stress; (bottom) vertical stress.

\section{CONCLUSION}

Point clouds can play a fundamental role in structural simulation of existing constructions, especially in the case complex historical structures. On the other hand, point clouds are just one of the input parameters behind the creation of BIM models with parametric intelligence and attributes. Building information modelling combines geometry with materials, construction phases, technological aspects, stratigraphic analysis, (...), and information from other inspections such as infrared thermography or structural tests.

It is clear that the creation of the BIM cannot be carried by using only the point cloud. The constructive logic must be incorporated into the model in order to understand the behaviour of the different structural elements. This could be intended as a new challenge for operators who work in the fields of photogrammetry, laser scanning, and 3D modelling. The use of the procedure based on curve networks (from 
clouds) and parametrization of NURBS surfaces (Oreni et al., $2014 \mathrm{~b}$ ) was used to obtain a BIM that preserves the complexity offered by laser clouds. Then, a rationalization of the BIM can be carried out to generate a robust and reliable finite element model for structural simulation.

The main aim of this work was to investigate the opportunity to generate such model by exploiting a preliminary accurate building information modelling. The work carried out is only the starting point: manual corrections were mandatory because of the complete lack of "intelligent" algorithms able to perform automated (i) point cloud $\rightarrow$ (ii) BIM $\rightarrow$ (iii) FEM conversion. Indeed, the conversion is not only limited to geometrical aspects. Additional architectural and structural considerations must be taken into account. From this point of view, much research is needed to combine geometric considerations with structural and architectural aspects.

\section{ACKNOWLEDGEMENTS}

This work was supported by the Interreg project "La Conservazione Programmata nello Spazio Comune Retico" (CPRE). The authors want to thank P. Crespi, N. Giordano and E. Rosina for the technical assistance concerning structural analysis and IR thermography. We are thankful to the staff of Municipality of Sondrio, in particular F. Barri, and Foppoli Moretta e Associati s.r.l. for providing several datasets used in this work.

\section{REFERENCES}

Binda, L., Tiraboschi, C., 1999. Flat-Jack Test: a slightly destructive technique for the diagnosis of brick and stone masonry structures. 8th International Conference and Exhibition, Structural Faults and Repair.

Brumana, R., Oreni, D., Cuca, B., Binda, L., Condoleo, P., Triggiani, M., 2014. Strategy for integrated surveing techniques finalized to interpretive models in a byzantine church, Mesopotam, Albania. International Journal of Architectural Heritage, 8, pages 886-924.

Colla, C., Largo, A., Corvaglia, P., Ubertini, F., 2008. Thermography investigations of roman archaeological masonry. In Binda, L., Di Prisco, M., Felicetti, R. editors, On Site Assessment of Concrete, Masonry and Timber Structures, SACoMaTiS 2008: Proceedings of the First International RILEM Symposium, volume 2, pages 923 - 932, Varenna, Italy, 1-2 September. RILEM Publications.

Della Torre, S., 2011. How the Concept of Preventive Conservation implemented on Built Cultural Heritage can work as a Factor for Regional Economic Development. In PRECOAH 2011 International Conference on Preventive Conservation of Architectural Heritage, pages 14 - 22, Nanjing (China), 29-30 October.

Eastman, C., Teicholz, P., Sacks, R., Liston, K., 2008. BIM Handbook - A guide to Building Information Modeling for owners, managers, designers, engineers, and contractors. John Wiley \& Sons, Inc..

Fai, S., Graham, K., Duckworth, T., Wood, N. and Attar, R., 2011. Building Information Modeling and Heritage Documentation. CIPA 2011 Conference Proceedings: XXIIIrd International CIPA Symposium, 8 pages.
Gusmeroli, G., Schiantarelli, G., 2014. From Laser Clouds to BIM and Finite Element Analysis: the Case Study of Castel Masegra. Master Thesis in Civil Engineering for Risk Mitigation, Politecnico di Milano, 138 pages.

Lee, G., Sacks, R., Eastman, C. M., 2006. Specifying parametric building object behaviour (BOB) for a Building Information Modeling system. Automation in Construction, 15(6):758-776.

Gregorczyk, P., Lourenço P. B., 2000. A review on Flat-Jack Testing. Engenharia Civil, (9):39 - 50.

Drogemuller, R., Steel, J., Toth, B., 2012. Model interoperability in Building Information Modelling. Software \& Systems Modeling, 11(1):99-109.

Oreni, D., Brumana, R., Cuca, B., Georgopoulos, A., 2013. HBIM for conservation and management of built heritage: Towards a library of vaults and wooden bean floors. In CIPA 2013 XXV International Symposium, ISPRS Annals, volume 164 , pages $1-6$.

Oreni, D., Brumana, R., Della Torre, S., Banfi, F., Barazzetti, L., Previtali, M., 2014a. Survey turned into HBIM: the restoration and the work involved concerning the Basilica di Collemaggio after the earthquake (L'Aquila). ISPRS Annals of the Photogrammetry, Remote Sensing and Spatial Information Sciences, vol.II, pages 267-273.

Oreni, D., Brumana, R., Banfi, F., Bertola, L., Barazzetti, L., Cuca, B., Previtali, M., Roncoroni, F., 2014b. Beyond Crude 3D Models: From Point Clouds to Historical Building Information Modeling via NURBS. In Digital Heritage. Progress in Cultural Heritage: Documentation, Preservation, and Protection, volume 8740, pages 166-175. Springer International Publishing.

Osello, A., 2012. The future of drawing with BIM for Engineers and Architects. Dario Flaccovio Editore s.r.l..

Piegl, L. A., Tiller, W., 1997. The NURBS book. Springer.

Piegl, L. A., Tiller, W., 1999. Computing offsets of NURBS curves and surfaces. Computer-Aided Design, 31(2):147-156.

Remondino, F., El-Hakim, S. F., Grün, A., Zhang, L., 2008. Turning images into 3-D models - Developments and performance analysis of images matching for detailed surface reconstruction of heritage objects. IEEE Signal Processing Magazine, 36(5):55-65.

Rosina, E., Grinzato, E., 2001. Infrared and Thermal Testing for Conservation of Historic Building. Material Evaluation, ASNT Journal, 59/n(8):942 - 954. ASNT Columbus (OH) USA.

Strang, G., Fix, G. J., 1973. An analysis of the finite element method.

Zienkiewicz, O. C., 1971. The finite element method in engineering science. McGraw-Hill. 